\title{
B Integrins Mediate FAK Y397 Autophosphorylation of Resistance Arteries during Eutrophic Inward Remodeling in Hypertension
}

\author{
Egidius H.J. Heerkens Lisa Quinn Sarah B. Withers Anthony M. Heagerty
}

Institute of Cardiovascular Sciences, University of Manchester, Manchester, UK

\section{Key Words}

Integrins · Resistance arteries · Eutrophic remodeling •

Hypertension

\begin{abstract}
Human essential hypertension is characterized by eutrophic inward remodeling of the resistance arteries with little evidence of hypertrophy. Upregulation of aV $\beta 3$ integrin is crucial during this process. In order to investigate the role of focal adhesion kinase (FAK) activation in this process, the level of FAK Y397 autophosphorylation was studied in small blood vessels from young TGR(mRen2)27 animals as blood pressure rose and eutrophic inward remodeling took place. Between weeks 4 and 5, this process was completed and accompanied by a significant increase in FAK phosphorylation compared with normotensive control animals. Phosphorylated (p)FAK Y397 was coimmunoprecipitated with both $\beta 1$ and $\beta 3$-integrin-specific antibodies. In contrast, only a fraction ( $<10$-fold) was coprecipitated with the $\beta 3$ integrin subunit in control vessels. Inhibition of eutrophic remodeling by cRGDfV treatment of TGR(mRen2)27 rats resulted in the development of smooth-muscle-cell hypertrophy and a signif-
\end{abstract}

icant further enhancement of FAK Y397 phosphorylation, but this time with exclusive coassociation of pFAK Y397 with integrin $\beta 1$. We established that phosphorylation of FAK Y397 with association with $\beta 1$ and $\beta 3$ integrins occurs with pressure-induced eutrophic remodeling. Inhibiting this process leads to an adaptive hypertrophic vascular response induced by a distinct $\beta 1$-mediated FAK phosphorylation pattern.

(c) 2014 S. Karger AG, Basel

\section{Introduction}

The resistance arteries ( $<300 \mu \mathrm{m}$ in internal diameter) are essential for mediating the autoregulation of flow and the stabilization of capillary pressure. An important component of this protective mechanism, called the myogenic response, allows these vessels to change diameter in response to alterations in intraluminar pressure. Besides resulting in enhanced myogenicity of the resistance arteries [1], sustained high blood pressure is associated with changes in cardiovascular structure. Resistance vessels develop a narrowed lumen and a thickened vascular wall,

\begin{tabular}{ll}
\hline KARGER & $\begin{array}{l}\text { ( 2014 S. Karger AG, Basel } \\
1018-1172 / 14 / 0514-0305 \$ 00.00 / 0\end{array}$ \\
E-Mail karger@karger.com & $\begin{array}{l}\text { This is an Open Access article licensed under the terms of } \\
\text { the Creative Commons Attribution 3.0 Unported license } \\
\text { (CC BY 3.0) (www.karger.com/OA-license-WT), appli- } \\
\text { cable to the online version of the article only. }\end{array}$
\end{tabular}

Dr. Sarah B. Withers

Institute of Cardiovascular Sciences

Core Technology Facility, University of Manchester

46 Grafton Street, Manchester M13 9NT (UK)

E-Mail sarah.withers@manchester.ac.uk 
but without an apparent increase in cross-sectional area. This process, known as inward eutrophic remodeling, occurs as a response to prolonged (myogenic) constriction in order to protect the downstream vasculature, and it is thought to be an energetically favored mechanism to preserve a reduced lumen diameter for long periods. Such a structural adaptation involves a migratory process, whereby vascular smooth-muscle cells (VSMCs) reposition in the vascular wall [2], and it can be observed in most forms of hypertension including onset and milder hypertensive states. When hypertrophy is observed, the rise in blood pressure is often fulminant and severe. Autoregulatory mechanisms are then inadequate to normalize wall stress. Recently, it has been reported that the development of small-artery hypertrophy is an adverse prognostic sign [3].

The integrin/extracellular-matrix (ECM) axis transfers tensile forces exerted by blood pressure across the cell membrane. Integrins are cell surface ECM receptors that can heterodimerize in a noncovalent fashion to form 24 different $\alpha \beta$ receptors which recognize a wide variety of cellular and ECM components present in the arterial extracellular space including collagen, fibronectin and laminin [4]. In resistance arteries, specific integrin subtypes are initially utilized for the mechanotransduction of pressure [5], while others mediate the migration of VSMCs towards a narrowed lumen $[6,7]$. Engagement of integrins upon migration activates intracellular signaling complexes found at focal adhesion (FA) sites [8]. To date, there has been no conclusive evidence that FA sites, which contain active tyrosine kinases (e.g. focal adhesion kinase, FAK) and other cytoskeletal associated phosphoproteins observed in migrating cells in vitro [9], exist in vivo in arterial VSMCs. However, it is clear that the migratory process of arterial VSMCs in vivo is more subtle and is limited to the elongation of tapered cells and an increase in cell overlap [2] compared with the more pronounced movement of cells in vitro. Eutrophic remodeling is a relatively rapid process that is followed by the fixation of cells within the vascular ECM scaffold and it requires active transglutaminases [10]. Recently, we described a key role for $\alpha \mathrm{V} \beta 3$ integrin in eutrophic remodeling and other studies have described its function in myogenic constriction [6].

There is increasing evidence that FAK is an important mediator for cell attachment and mechanosensing [11]. The Src family kinases are also regarded as key components of mechanotransduction to special domains via the cytoskeleton when force is applied to fibronectin-binding integrins such as $\alpha \mathrm{V} \beta 3$ [12]. Moreover, FAK/Src associa- tion with specific integrin subunits seems to regulate particular cellular functions: for example, $\alpha 5 \beta 1$ appears to regulate the L-type calcium current, which mediates myogenic constriction via Src and FAK signaling [13], whereas the association of FAK/Src with $\beta 3$ integrin subunits seems to be important for cell migration [14]. In addition, evidence is accumulating for the initiation of FAK phosphorylation in the growth response of arteries exposed to elevated intraluminal pressure [15].

We wished to test the hypothesis that FAK/Src signaling is crucial in mechanotransduction and the eutrophic inward remodeling of resistance arteries as a response to elevated pressure.

This study examined the activation levels of FAK/Src in arteries exposed to elevated pressure in vitro and ex vivo, and we report differential FAK Y397 phosphorylation levels present at the integrin $\beta$ subunits of resistance arteries from hypertensive TGR(mRen2)27 rats as pressure rises and eutrophic inward remodeling occurs. Ex vivo pressurization of arterial segments which exhibit myogenic tone confirmed that FAK Y397 phosphorylation is increased as a response to elevated pressure.

\section{Methods}

Animals

The TGR(mRen2)27 rat carries a DBA/2J Ren2 transgene and develops hypertension with vascular structural changes from eutrophic inward remodeling $[6,16]$. These animals, together with Sprague-Dawley (SD) normotensive control animals, were studied by immunohistochemistry and Western blot at 4 and 5 weeks of age. Ex vivo studies were performed on arteries harvested from SD rats at 5 weeks of age. On the day of study, rats were sacrificed by stunning and cervical dislocation. All animal procedures were performed in accordance with the UK Home Office Regulated Procedures on Living Animals (Scientific Procedures) Act 1986.

\section{Blood Pressure Measurement}

Blood pressure measurements of TGR(mRen2)27 and SD control animals were performed using tail-cuff plethysmography under light fluothane anesthesia.

\section{Vessel Morphology and Integrin Antagonism}

We performed studies on the small arteries harvested from animals sacrificed at 5 weeks of age, because hypertension-induced eutrophic remodeling takes place from the age of 4 weeks and is completed by week 5 , as described previously [6]. Segments of 2 ndorder arteries (approx. $200 \mu \mathrm{m}$ ) were isolated from the proximal region of the mesenteric bed, mounted on a wire myograph and held in physiological salt solution (PSS; $119 \mathrm{mM} \mathrm{NaCl}, 4.7 \mathrm{mM} \mathrm{KCl}$, $25 \mathrm{mM} \mathrm{NaHCO}_{3}, 1.17 \mathrm{mM} \mathrm{KH}_{2} \mathrm{PO}_{4}, 1.17 \mathrm{mM} \mathrm{MgSO}_{4}, 0.026 \mathrm{~mm}$ EDTA, $1.6 \mathrm{mM} \mathrm{CaCl}_{2}$ and $5.5 \mathrm{mM}$ glucose) at $37^{\circ} \mathrm{C}$ and gassed with $5 \% \mathrm{CO}_{2}$ and $95 \% \mathrm{O}_{2}$ to maintain a $\mathrm{pH}$ of 7.4. Subsequent morpho- 
logical parameters of live vessels such as the media-to-lumen ratio, growth and remodeling indices were then measured and calculated, as previously described [17].

In order to study the effects of integrin $\alpha \mathrm{V}$ antagonism, TGR(mRen2)27 rats were injected intraperitoneally at the age of 4 weeks with cRGDfV or cRADfV peptide $(10 \mathrm{mg} / \mathrm{kg}$; Calbiochem) twice daily for 5 days before sacrifice [6]. These act as an $\alpha \mathrm{V}$ integrin-specific inhibitory peptide, thereby inhibiting integrin functions or inactive control peptide, respectively [18-20]. Arteries were then harvested for morphological measurements using wire myography.

\section{Phosphorylated FAK Y397 Western Blot Analysis}

Western blot analysis was performed according to the methodology described by Laemmli [21]. Vessels were dissected and protein extracted on ice in radioimmunoprecipitation assay buffer containing phosphatase and protease inhibitors to prevent changes in the phosphorylation status of the proteins under investigation. Anti-phosphorylated (p)FAK Y397 antibody (1:1,000, $0.5 \mu \mathrm{g} /$ $\mathrm{ml}$; AB4803, Abcam, Nottingham, UK), with a total (pan)-FAK antibody as a control for loading $(1: 1,000,1.0 \mu \mathrm{g} / \mathrm{ml}$; AB1311, Abcam), was used to detect expression in $25 \mu \mathrm{g}$ of total protein. pFAK Y397 expression levels were corrected for the amount of total (pan)-FAK. Densitometric analysis was performed on a BioRadGS690 scanner.

\section{Integrin $\alpha V$ Immunoprecipitation}

An amount of $5 \mu \mathrm{g}$ of each antibody was used to precipitate integrin subunits from $250 \mu \mathrm{g}$ of total resistance artery radioimmunoprecipitation assay extract. The antibodies used for immunoprecipitation were: $\beta 1$ (AB1952), $\beta 3$ (AB1932) and $\alpha 5$ (aba5b) integrin antibodies (Chemi-Con, Moses Lake, Wash., USA). Agarose IgG/protein A (Sigma-Aldrich, UK) was used to bind and identify coprecipitated complexes. Subsequent Western blot analysis was performed using FAK Y397 (1:1,000) antibody and Src Y418 (1:500, $0.5 \mu \mathrm{g} / \mathrm{ml}$; Abcam). Protein A-HRP was used for chemiluminescent detection of coprecipitated proteins [22].

\section{Ex vivo Pressure Arteriography}

Arterial segments from the proximal region of the cremasteric artery from SD animals were dissected and mounted between two glass microcannulae in physiological salt solution in a pressure myograph (Living Systems Instrumentation, Burlington, Vt., USA). Experiments were carried out at $32^{\circ} \mathrm{C}$. PP2 and PP3, i.e. a potent inhibitor of $\mathrm{c}$-Src and an inactive analog (negative control) of PP2, respectively $[23,24]$, were used at $1 \mu \mathrm{M}$ (Calbiochem, Cambridge, UK). Only arteries that exhibited pressure-mediated constriction were included in the study. After pressurization at either 60 or $120 \mathrm{~mm} \mathrm{Hg}$ for $1 \mathrm{~h}$, arteries were quickly fixed in ice-cold $4 \%$ paraformaldehyde solution, left overnight, washed in $70 \%$ ethanol and prepared for histology/immunofluorescence. Immunohistochemical preparations of arteries ex vivo and in vitro were the same.

\section{Immunofluorescence Localization}

Vessels for immunofluorescence were dissected on ice and snap-fixed in ice-cold $4 \%$ paraformaldehyde. Paraffin-embedded arteries were sliced at $4 \mu \mathrm{m}$, dewaxed and rehydrated. The pFAK Y397 integrin (1:100; AB4803, Abcam) and a nonimmune control IgG antibody were used separately, followed by donkey anti-rabbit
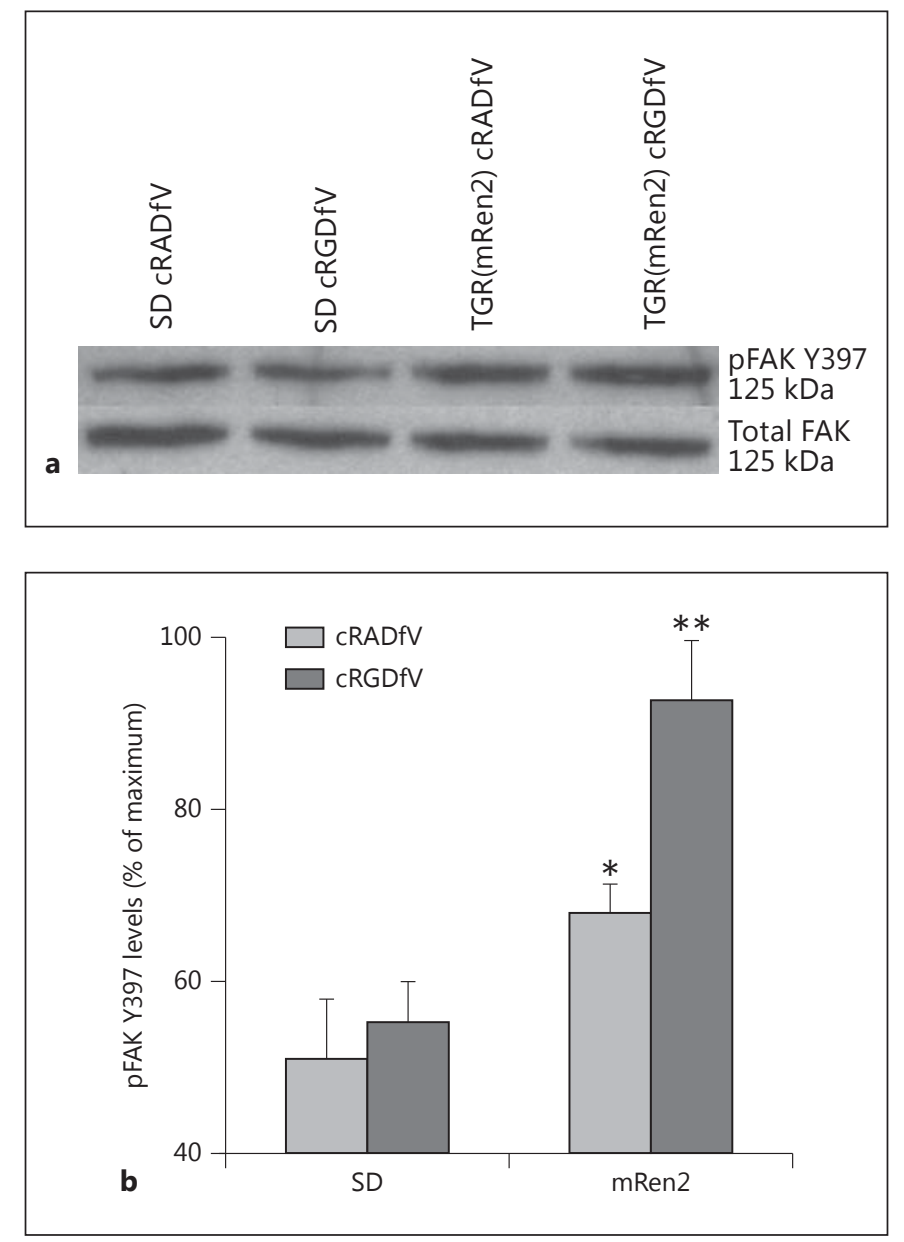

Fig. 1. FAK Y397 phosphorylation in the resistance arteries of TGR(mRen2)27 rats treated with cRGDfV. a Representative Western blot of pFAK Y397 and total FAK (125 kDa) using $25 \mu \mathrm{g}$ of protein from resistance artery extracts. b Levels of pFAK Y397 analyzed by densitometric scans and corrected for total FAK ( $\mathrm{n} \geq$ $\left.4,{ }^{*} \mathrm{p}<0.05,{ }^{* *} \mathrm{p}<0.01\right)$.

antibody with conjugated TRITC (1:200, $1 \mu \mathrm{g} / \mathrm{ml}$; Jackson ImmunResearch Laboratories, West Grove, Pa., USA). Diamidino2-phenylindole (DAPI, 1 pg/ml) (Jackson ImmunoResearch Laboratories) was used as a blue fluorescent nuclear stain. A Leica DM6000 epifluorescence microscope was used at $\times 1,000$ magnification in conjunction with Leica FW4000 software for image capture and analysis. Superimposing fluorescence and phase-contrast microscopy images allowed the visualization of the VSMC membrane and the internal elastic lamina which indicates the smoothmuscle/endothelium boundary.

\section{Statistical Analysis}

Statistical analysis was performed using MS Excel. Values are expressed as mean \pm standard deviation. Differences between data were tested using the two-tailed unpaired homoscedastic Student $\mathrm{t}$ test. $\mathrm{p}<0.05$ was considered statistically significant. 
Fig. 2. Basal levels of pFAK Y397 immunofluorescence in resistance mesenteric arteries. a, c TGR(mRen2)27 rat mesenteric artery and the prominent localization of pFAK Y397 to VSMCs. b, d pFAK Y397 localization in $\mathrm{SD}$ rat resistance arteries is loosely distributed throughout the cells in contrast with TGR(mRen2)27 rat arteries. $\times 1,000$. Scale bar: $25 \mu \mathrm{m}$.
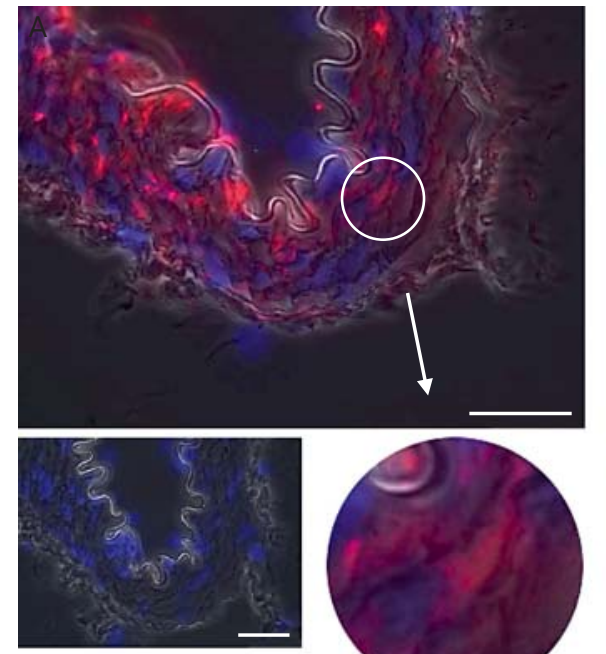

a

Blank

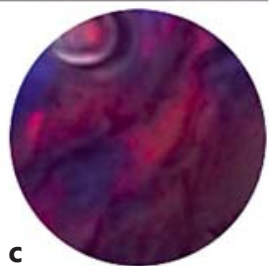

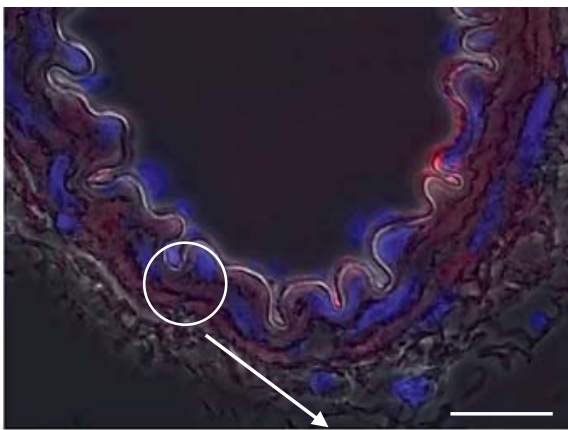

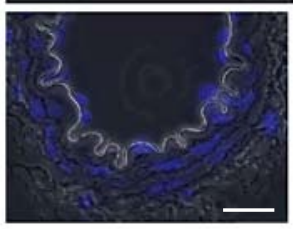

b

Blank

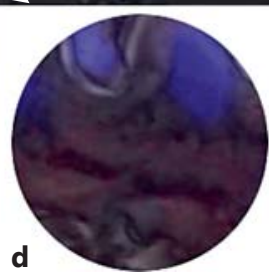

\section{Results}

\section{Blood Pressure}

Between 4 and 5 weeks of age, mean systolic blood pressure in TGR(mRen2)27 rats was significantly increased from $121 \pm 7.9$ to $148 \pm 3.0 \mathrm{~mm} \mathrm{Hg}(\mathrm{n} \geq 6, \mathrm{p}<0.02)$. SD control rats showed no age-dependent increase in blood pressure which ranged from $80.9 \pm 8.8$ to $93.4 \pm 8.1 \mathrm{~mm} \mathrm{Hg}$. Pressures in TGR(mRen2)27 rats were significantly higher than those in SD rats at both time points $(\mathrm{p}<0.0001)$.

The administration of $c R G D f V$ and $c R A D f V$ peptides had no effect on the blood pressure rise seen in TGR(mRen2)27 rats (data not shown).

\section{Vascular Morphology}

The cross-sectional area of TGR(mRen2)27 rat arteries at 5 weeks was unchanged compared with at 4 weeks of age, with a remodeling index of $97 \%$ indicating the development of eutrophic inward remodeling due to the media-to-lumen ratio being significantly increased $(\mathrm{p}<$ 0.01 ). The structural parameters of SD rat arteries did not change over 7 days.

cRGDfV treatment reduced the remodeling index to 9\% and there was evidence of hypertrophic growth, with a calculated growth index of $17 \%$. Treatment with cRADfV had no effect on remodeling, as predicted (data not shown).

\section{Western Blot}

Western blot analysis of pFAK Y397 showed significant rises in autophosphorylation levels in resistance arteries from TGR(mRen2)27 animals treated with
cRADfV control peptide (approx. 1.4-fold, $\mathrm{p}<0.05$ ), compared with vessels from SD normotensive control animals (fig. 1a, b). Surprisingly, an increase in pFAK of approximately 2 -fold was observed in TGR(mRen2) 27 rat arteries treated with the active cRGDfV peptide $(\mathrm{p}<0.01)$ compared with vessels from normotensive animals treated with the same peptide $(n \geq 4)$. Relative pFAK Y397 levels, established by densitometry, were corrected by comparison to total (pan)-FAK (fig. 1a, b).

\section{Immunofluorescence}

Phospho-FAK Y397 immunofluorescent staining of the arteries from normotensive SD animals was faint but present throughout the medial smooth-muscle cells ( $\mathrm{n} \geq 3$; fig. 2b, d). The level of pFAK Y397 was observed to be increased in the VSMCs of TGR(mRen2)27 rat ( $\mathrm{n} \geq$ 3) resistance arteries (fig. 2a). Superimposing phase-contrast images onto fluorescent signals allowed the visualization of the VSMC medial layer, the internal elastic lamina and intense fluorescent pFAK Y397 regions of staining present in TGR(mRen2)27 rat VSMCs (fig. 2c). This is in contrast with fluorescence of total (nonphosphorylated) FAK (fig. 3a-d), which is expressed diffusely throughout the VSMCs of the medial layer (fig. 3c). FAK was localized to FA sites as identified by coimmunohistochemistry for FAK Y397 and vinculin, a membrane protein found associated with the FA sites of the elastic lamina (fig. 4). Control incubations with nonimmune IgG did not result in any detectable signal (fig. 2-4). The treatment of animals with the cRGDfV peptide $(10 \mathrm{mg} / \mathrm{kg})$ showed localization of pFAK within the medial layer of 
Fig. 3. Total FAK (nonphosphorylated) immunofluorescence in resistance arteries. a, c The localization of total FAK throughout the VSMCs of TGR(mRen2)27 rat mesenteric artery. b, d Similarly, total FAK is present throughout the VSMCs of SD rat mesenteric arteries. $\times 1,000$. Scale bar: 25 $\mu \mathrm{m}$.

Fig. 4. Localization of FAK Y397 at FA sites in SD rat resistance arteries. a DAPI staining for nuclei of within the resistance artery. FAK Y397 (b), vinculin staining (c) and phase-contrast images of the elastic lamina (d) demonstrate colocalization of FAK Y397 to FA sites within the medial layer of the artery (e). $\mathbf{f}$ A blank control.
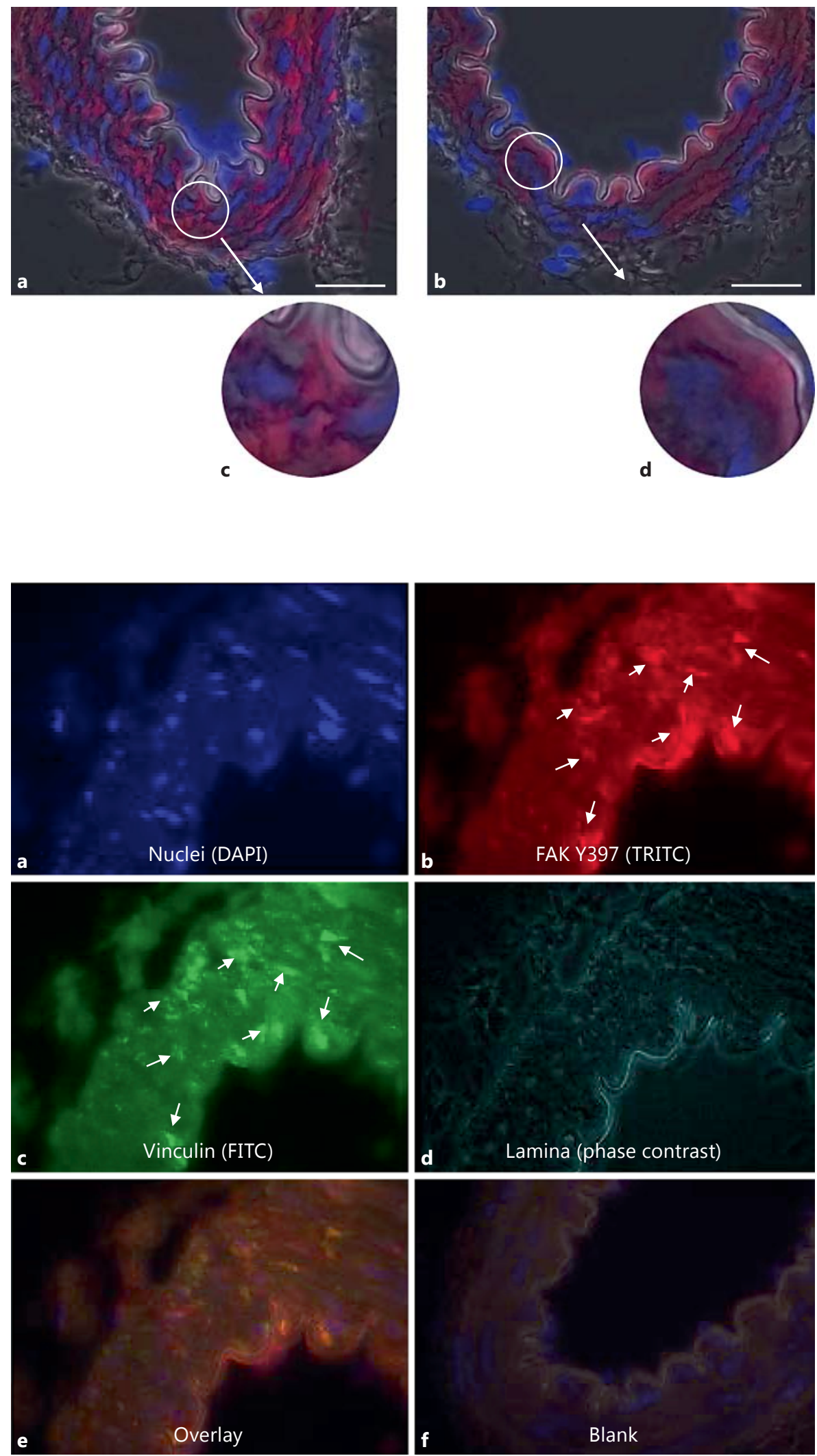
Fig. 5. pFAK Y397 immunofluorescence of rat mesenteric arteries following $\alpha 5$ antagonism by cRGDfV treatment $(10 \mathrm{mg} / \mathrm{kg}$, intraperitoneal injection twice daily for 5 days). a, c pFAK Y397 staining is increased and present throughout the VSMC medial layer of TGR(mRen2)27 rat resistance arteries. $\mathbf{b}, \mathbf{d}$ In contrast, cRGDfV treatment has no effect on pFAK Y397 levels in SD rat mesenteric arteries. $\times 1,000$. Scale bar: 25 $\mu \mathrm{m}$.
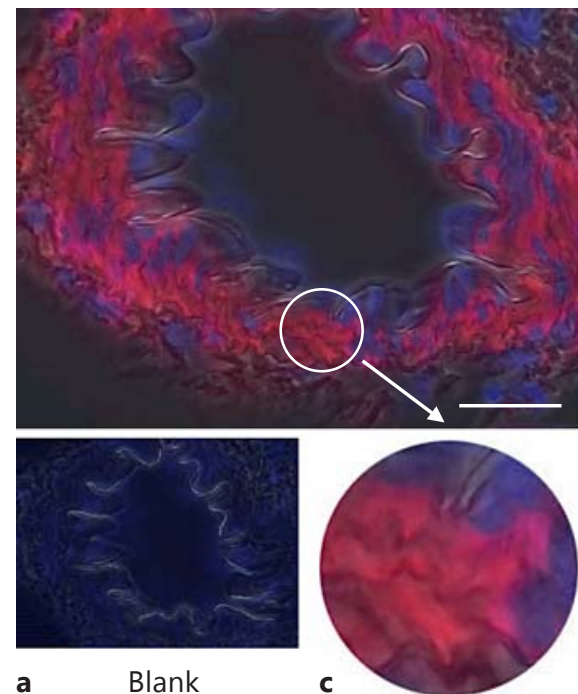

c

\begin{abstract}
a Blank
\end{abstract}

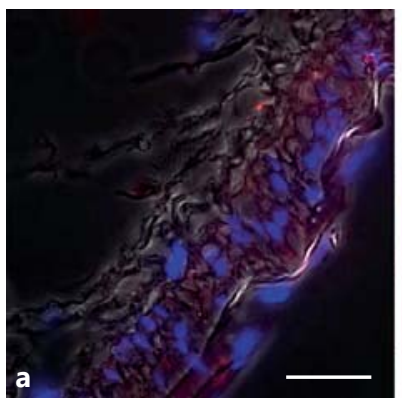

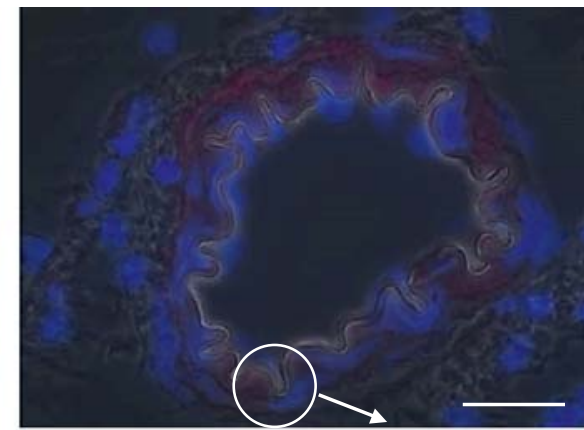

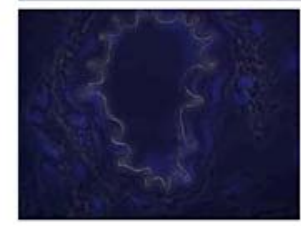

Blank

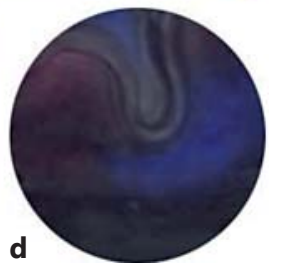

5 phosphorylated FAK Y397 after ex vivo pressurization (60 and $120 \mathrm{~mm} \mathrm{Hg}$ ) of cremaster arteries. a, b PP3 coincubation has no effect on pressure-induced myogenic constriction or phosphorylation levels of FAK Y397. a Arteries at $60 \mathrm{~mm} \mathrm{Hg}$ only present minimal phosphorylation of FAK Y397. b Arteries pressurized at $120 \mathrm{~mm} \mathrm{Hg}$ exhibit prominent phosphorylation levels of FAK Y397. c Phosphorylation of FAK Y397 was abrogated when arteries were incubated with $1 \mu \mathrm{M}$ PP2. Scale bar: $25 \mu \mathrm{m}$.

VSMCs and more intense staining in the treated TGR(mRen)27 rat arteries than in those from SD rats $(\mathrm{n} \geq 3$; fig. 5a) and more diffuse than in untreated arteries (fig. 2a), thereby supporting quantified data from our observations from Western blot analysis (fig. 1). Figure 5 a, c clearly shows intense Y397 staining is throughout the medial layer of arteries. In contrast, cRGDfV treatment of normotensive SD animals did not have any effect on pFAK Y397 levels (fig. 5b, d).

Inspection of arterial segments undergoing FAK Y397 phosphorylation staining, which were pressurized at 120 $\mathrm{mm} \mathrm{Hg}$ for $1 \mathrm{~h}$ (and then developed myogenic-tone ex vivo) exhibited increases in FAK Y397 phosphorylation compared with vessels pressurized at $60 \mathrm{~mm} \mathrm{Hg}(\mathrm{n} \geq 5)$. No passive morphological alterations of vessels to pressure were observed at this stage (data not shown). Phosphorylation of FAK Y397 at $120 \mathrm{~mm} \mathrm{Hg}$ was abrogated when arteries were coincubated with $1 \mu \mathrm{M}$ of PP2. In contrast, coincubation of PP3 did not have any effect (fig. 6).

\section{Integrin Coimmunoprecipitation}

pFAK Y397 was coprecipitated with integrin $\beta 3$ and, to a lesser extent, with integrin $\alpha 5 \beta 1$ (fig. $7 a$ ) from the protein extracts of SD rat arteries. However, no FAK was coprecipitated with the general subpopulation of $\beta 1$ integrins (fig. 7a).

In contrast to the protein extracts of normotensive SD resistance arteries, pFAK Y397 coprecipitation with all the integrins, i.e. $\beta 1, \beta 3$ and $\alpha 5 \beta 1$, was observed in the arteries of TGR(mRen2)27 animals (fig. 7b). Coimmunoprecipitation of the protein extracts from resistance arteries of 5-week-old cRGDfV-treated TGR(mRen2)27 animals (which inhibited eutrophic inward remodeling but showed hypertrophy) did not show pFAK cobinding with $\beta 3$ or $\alpha 5 \beta 1$ integrins, but interacted solely with other subpopulations of $\beta 1$ integrins (fig. 7c).

In conjunction with $\mathrm{FAK}, \mathrm{c}-\mathrm{Src}$ was prominently coprecipitated with integrin $\alpha 5 \beta 1$ and, to a lesser extent, with integrin $\beta 3$ (fig. 8a). In contrast, c-Src is not coprecipitated with the $\alpha 5 \beta 1$ integrins of resistance arteries of 
Fig. 7. Integrin immunoprecipitation: coprecipitation of pFAK Y397. a pFAK Y397 is coprecipitated with $\beta 3$ and $\alpha 5 \beta 1$ integrins in SD rat arteries. b In contrast, pFAK Y397 is coprecipitated with all the integrins tested in TGR(mRen2)27 rat arteries. c Inhibition of remodeling with cRGDfV peptides results in coprecipitation of pFAK Y397 only with $\beta 1$ integrins. Immunoprecipitations shown are representative of at least 3 experiments.

Fig. 8. Integrin immunoprecipitation: coprecipitation of pSrc Y418. a pFAK pSrc Y418 is coprecipitated with $\beta 3$ and a5 $\beta 1$ integrins in SD rat arteries. b In TGR(mRen2)27 rats, pSrc Y418 is coprecipitated with $\beta 1$ and $\beta 3$ integrins. $\mathbf{c}$ In contrast, cRGDfV treatment results in coprecipitation of $\mathrm{pSrc}$ Y418 with $\beta 1$ integrins only. Immunoprecipitations shown are representative of at least 3 experiments.
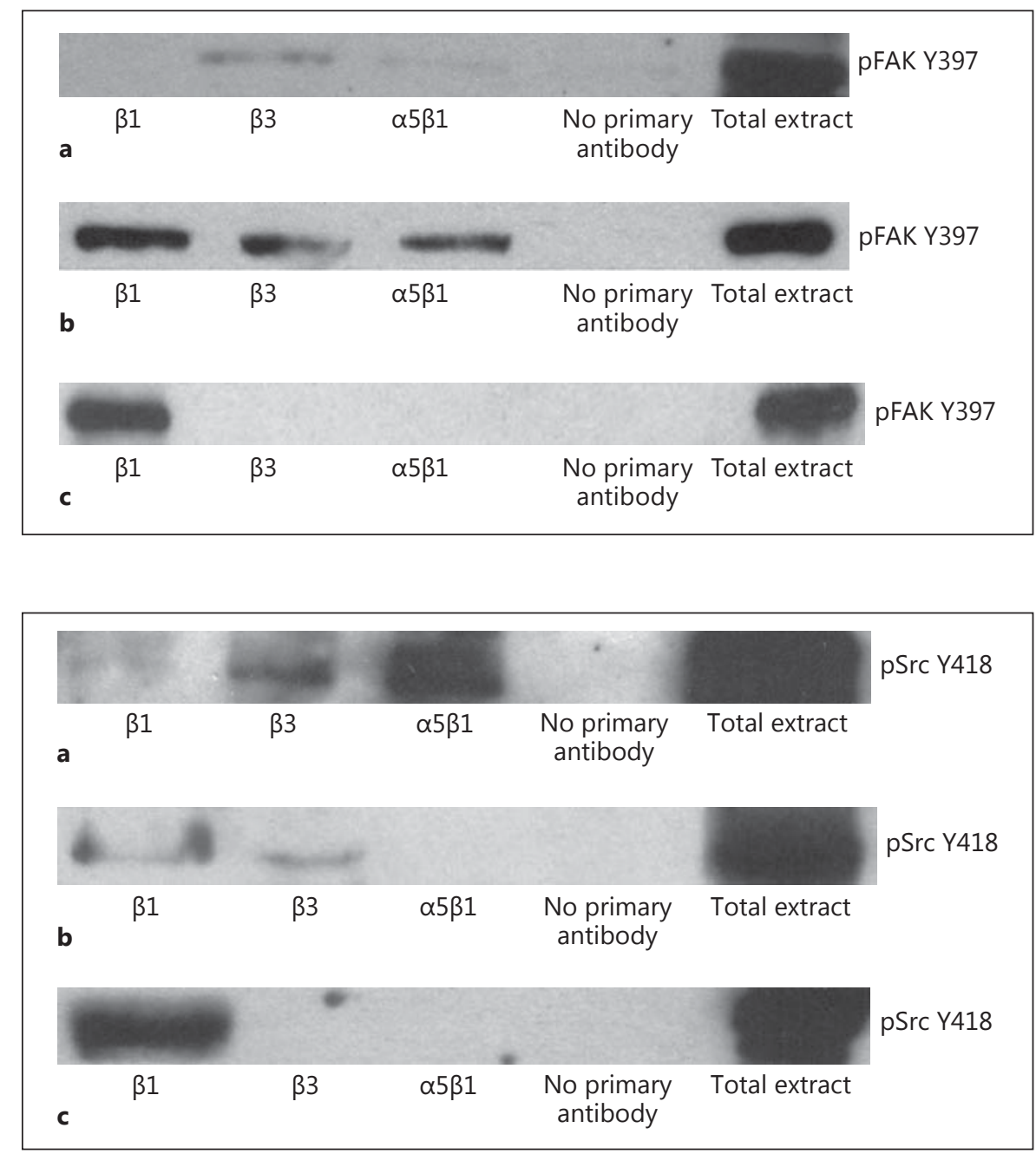

hypertensive TGR(mRen2)27 animals, but with integrins $\beta 1$ and $\beta 3$ (fig. 8 b). c-Src, however is not recruited to the $\beta 3$ cytoplasmic domain when $\alpha \mathrm{V} \beta 3$ binding to the ECM with the cRGDfV peptide is inhibited (fig. 8c).

\section{Discussion}

Previously, we showed that the hypertension-mediated inward eutrophic remodeling of TGR(mRen2)27 rat resistance arteries commenced at 4 weeks of age and was complete at 5 weeks [6]. This process was dependent on the integrin $\alpha \mathrm{V} \beta 3$ (the only VSMC $\beta 3$ integrin) because administration of cRGDfV peptide, which preferentially interferes with the binding of this integrin-heterodimer to the ECM [18-20, 25], prevented the remodeling process but enhanced hypertrophy. We also investigated the localization of FAK and its activation status in the smoothmuscle cells of TGR(mRen2)27 rat resistance arteries and determined its association with $\beta 1$ or $\beta 3$ integrin subunits.

We established that low basal levels of FAK Y397 phosphorylation in normotensive SD rat mesenteric arteries are predominantly associated with the $\beta 3$ integrin subunit and that active c-Src associates with integrin $\alpha 5 \beta 1$. FAK Y397 phosphorylation was significantly increased in hypertensive TGR(mRen2)27 resistance arteries and recruited to FA sites after blood pressure rose and once eutrophic inward remodeling was complete. In addition, both pFAK Y397 and active c-Src associated with $\beta 1$ and $\beta 3$ integrins in the resistance arteries of TGR(mRen2)27 animals. However, the inhibition of remodeling by the administration of cRGDfV peptides resulted in the hypertrophy of VSMCs and a further increase of FAK Y397 


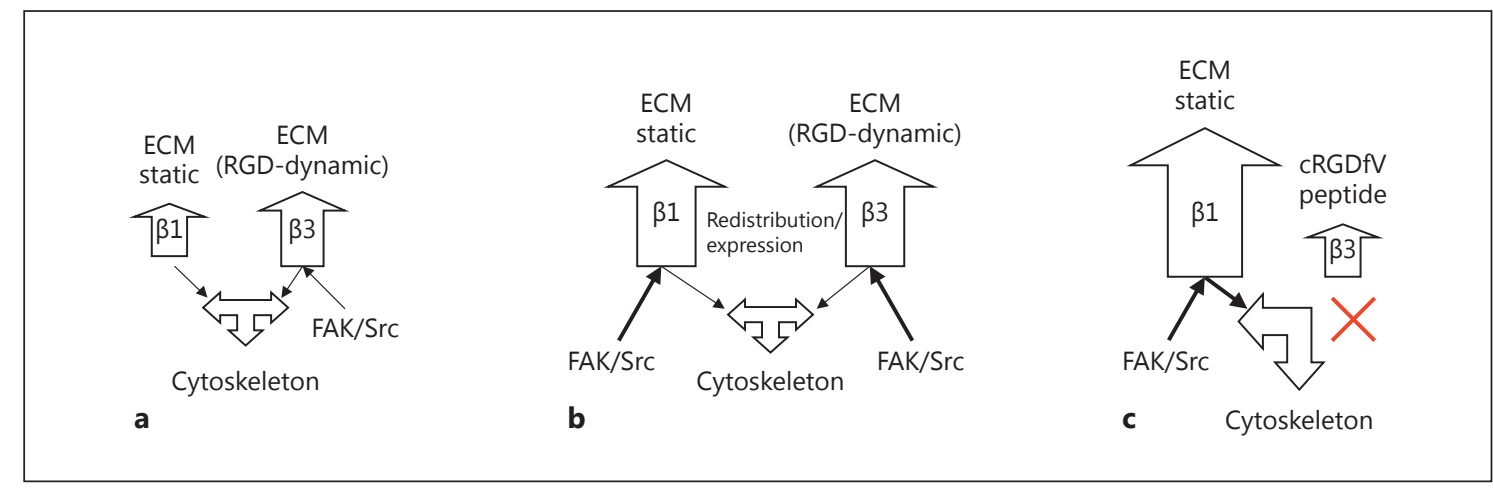

Fig. 9. Schematic representation of the role of $\beta$ integrins in mediating FAK Y397 autophosphorylation of the resistance arteries. a Normotenisve: integrin $\alpha 5 \beta 1$ (involved in calcium influx) and $\beta 3$ integrin subunits are mechanically activated resulting in FAK Y397/Src Y418 phosphorylation, and are probably required for normal vascular integrity during normotensive pressure fluctuations. Relatively little change in FAK Y397 and Scr Y418 phosphorylation is required for normal vascular adaptations to minor changes in pressure. Signaling of FAK/Src via 'general' ECM-anchoring $\beta 1$ integrins is minimal. b Hypertensive inward remodeling with cRADfV: during hypertensive remodeling, an increase in phosphorylation of the mechanosensitive FAK Y397/Src Y418 sig-

phosphorylation. This process was associated with integrins containing the $\beta 1$ subunit only.

We can conclude from these data that FAK phosphorylated at tyrosine position 397 (the autophosphorylation site) is present at both the integrin $\beta 1$ and $\beta 3$ cytoplasmic sites in the resistance arteries of hypertensive TGR(mRen2)27 animals, in contrast to SD controls, which exhibit relatively low pFAK Y397 levels only associated with the integrin $\beta 3$ subunit.

We cannot rule out the possible involvement of FAK Y397 phosphorylation in subsequent length/autoregulation processes during the hypertension remodeling processes downstream of the integrin $\beta 3$ subunit. However, it is now thought that the initial role, in the light of the FAK Y397 phosphorylation observed, is in mechanotransduction, as cRGDfV peptides not only inhibited eutrophic remodeling but also encouraged further FAK Y397 phosphorylation during the onset of hypertension in this model. Therefore, the increase of FAK Y397 phosphorylation observed is more likely to occur as a consequence of increases in wall tension, rather than acting as a direct intermediate of eutrophic inward remodeling. Besides aiding adhesion and migration [26], $\beta 1$ and $\beta 3$ integrins are both responsible for the intracellular transfer of these forces in the VSMCs of resistance arteries [27, 28]. This conclusion is strengthened further naling complex is mediated via $\beta 1$ and $\beta 3$ integrins (but not the pSrc Y418 component of $\alpha 5 \beta 1$ ), suggesting a role of $\beta 1$ and $\beta 3$ integrin subunits to maintain vascular integrity in response to an increase in pressure. c Hypertrophic remodeling: abrogation of $\alpha \mathrm{V} \beta 3$ signaling with cRGDfV results in hypertrophy in response to pressure and mechanosignaling exclusively through $\beta 1$ integrins. The majority of the $\beta 1$ integrins are thought to be important in 'anchoring' VSMCs within the matrix of the vascular wall. Exclusive mechanosensing of FAK Y 397 via $\beta 1$ integrins through this rigid scaffold is thought to be central to these structural adaptations in hypertension.

by how the arterial segments of the cremaster muscle, at a high pressure (120 $\mathrm{mm} \mathrm{Hg}$ ), exhibit a prompt induction of FAK Y397 phosphorylation $(<1 \mathrm{~h})$ when compared with vessels pressurized at much lower levels and completely independent of neurohumoral influences [29].

There is the possibility that the increase of FAK Y397 phosphorylation observed in the resistance arteries of the young TGR(mRen2)27 hypertensive animals was induced by enhanced activity of the renin-angiotensin system (RAS; reviewed in [30]). However, this seems unlikely because at 5 weeks of age the resistance arteries of these animals have eutrophically remodeled without evidence of hypertrophy [6], similar to the morphological adaptations observed in a low-renin model of hypertension [31] and the BMH-2 mouse model which develops hypertension independently of the RAS [32]. The hallmark of a locally activated vascular RAS in older animals is vascular hypertrophy [30, 33]. Again, ex vivo pressurization has determined that FAK Y397 phosphorylation is likely to be associated with an increase in pressure, in an Src-dependent manner, without the possible interference of neurohumoral influences. However, we cannot completely rule out the contribution of RAS activity to FAK Y397 phosphorylation in the TGR(mRen2)27 resistance arteries [34, 35]. 
Various studies have reported the association of FAK with different $\beta$ integrin subunits; $\beta 1, \beta 3$ and $\beta 5$ cytoplasmic domains all contain sufficient information to trigger FAK phosphorylation and these specific regions have been identified [36]. Different cellular systems in vitro utilize different integrins for FAK activation, e.g. $\beta 3$ - but not $\beta 1$-integrin engagement in cultured cardiomyocytes and VSMCs is accompanied by FAK activation in FAs $[14,37]$. In contrast, fibroblasts cultured on collagen employ $\beta 1$ integrins only for FAK activation [38]. In response to the hepatocyte growth factor, VSMCs are known to utilize both $\beta 1$ and $\beta 3$ integrins for FAK activation and subsequent migration [39]. In this study, using the TGR(mRen2)27 rat model [16], it became apparent that both $\beta 1$ and $\beta 3$ integrins are involved in VSMC FAK recruitment/signaling when pressure becomes elevated, in contrast to normotensive controls. However, the FAK Y397 phosphorylation observed which is associated with $\beta$-integrin subunits in response to elevated pressure is not exclusively increased when eutrophic remodeling processes take place, but is already apparent at the initial stages when pressure becomes elevated.

\section{Clinical Perspectives}

It has been established that both $\beta 1$ and $\beta 3$ integrin subtypes are required not only in the adhesion of VSMCs to the ECM of the vascular wall but also for pressure-me- diated signaling, resulting in vascular myogenic constriction and remodeling in hypertension $[6,27]$ (summarized in fig. 9). In this study, we identified FAK Y397 phosphorylation as an early event as a result of elevated pressure in vivo and ex vivo. The identification of FAK Y397 phosphorylation in an Src-dependent manner is an important first step in resolving the complex signaling cascades that underlie integrin-mediated vascular adaptations of resistance arteries in hypertension. The relevance of unraveling molecular components in specific signaling events, in the context of pressure mechanotransduction in hypertension, will enlighten the design of pharmacological agents to prevent hypertension-mediated targetorgan damage.

Recent data have suggested that the morphological changes in the arterial wall of small blood vessels are associated with cardiovascular prognosis. The normal response to untreated hypertension is eutrophic inward remodeling, i.e. a rearrangement of the vascular wall architecture without any evidence of growth [17]. When hypertrophy supervenes, this heralds the breakdown of normal homeostatic mechanisms such as autoregulation and, as such, there is clear evidence that this is the structural alteration associated with an adverse prognosis [3].

\section{Acknowledgements}

This study was funded by the Wellcome Trust. We thank Mrs. Maureen Speed for her expert secretarial assistance.

\section{References}

1 Miller FJ, Jr, Dellsperger KC, Gutterman DD: Myogenic constriction of human coronary arterioles. Am J Physiol 1997;273:H257$\mathrm{H} 264$.

2 Martinez-Lemus LA, Hill MA, Bolz SS, Pohl U, Meininger GA: Acute mechanoadaptation of vascular smooth muscle cells in response to continuous arteriolar vasoconstriction: implications for functional remodeling. FASEB J 2004;18:708-710.

-3 Izzard AS, Rizzoni D, Agabiti-Rosei E, Heagerty AM: Small artery structure and hypertension: adaptive changes and target organ damage. J Hypertens 2005;23:247-250.

-4 Humphries MJ, Travis MA, Clark K, Mould AP: Mechanisms of integration of cells and extracellular matrices by integrins. Biochem Soc Trans 2004;32:822-825.
5 Martinez-Lemus LA, Sun Z, Trache A, Trzciakowski JP, Meininger GA: Integrins and regulation of the microcirculation: from arterioles to molecular studies using atomic force microscopy. Microcirculation 2005;12:99-112.

- 6 Heerkens EH, Shaw L, Ryding A, Brooker G, Mullins JJ, Austin C, et al: alphaV integrins are necessary for eutrophic inward remodeling of small arteries in hypertension. Hypertension 2006;47:281-287.

7 Slepian MJ, Massia SP, Dehdashti B, Fritz A Whitesell L: Beta3-integrins rather than beta1integrins dominate integrin-matrix interactions involved in postinjury smooth muscle cell migration. Circulation 1998;97:1818-1827.

8 Schlaepfer DD, Hunter T: Evidence for in vivo phosphorylation of the Grb2 SH2-domain binding site on focal adhesion kinase by Srcfamily protein-tyrosine kinases. Mol Cell Biol 1996;16:5623-5633.
9 Mitra SK, Hanson DA, Schlaepfer DD: Focal adhesion kinase: in command and control of cell motility. Nat Rev Mol Cell Biol 2005;6: 56-68.

10 Eftekhari A, Rahman A, Schaebel LH, Chen $\mathrm{H}$, Rasmussen CV, Aalkjaer C, et al: Chronic cystamine treatment inhibits small artery remodelling in rats. J Vasc Res 2007;44:471482.

11 Hynes RO: Cell adhesion: old and new questions. Trends Cell Biol 1999;9:M33-M37.

$>12$ Wang Y, Botvinick EL, Zhao Y, Berns MW, Usami S, Tsien RY, et al: Visualizing the mechanical activation of Src. Nature 2005;434: 1040-1045.

13 Wu X, Davis GE, Meininger GA, Wilson E, Davis MJ: Regulation of the L-type calcium channel by alpha 5beta 1 integrin requires signaling between focal adhesion proteins. J Biol Chem 2001;276:30285-30292. 
14 Willey CD, Balasubramanian S, Rodriguez Rosas MC, Ross RS, Kuppuswamy D: Focal complex formation in adult cardiomyocytes is accompanied by the activation of beta3 integrin and c-Src. J Mol Cell Cardiol 2003;35: 671-683.

$>15$ Rice DC, Dobrian AD, Schriver SD, Prewitt RL: Src autophosphorylation is an early event in pressure-mediated signaling pathways in isolated resistance arteries. Hypertension 2002;39:502-507.

-16 Mullins JJ, Peters J, Ganten D: Fulminant hypertension in transgenic rats harbouring the mouse Ren-2 gene. Nature 1990;344:541544.

$>17$ Heagerty AM, Aalkjaer C, Bund SJ, Korsgaard N, Mulvany MJ: Small artery structure in hypertension. Dual processes of remodeling and growth. Hypertension, 1993;21:391-397.

-18 Bayless KJ, Salazar R, Davis GE: RGD-dependent vacuolation and lumen formation observed during endothelial cell morphogenesis in three-dimensional fibrin matrices involves the alpha(v)beta(3) and alpha(5)beta(1) integrins. Am J Pathol 2000;156:1673-1683.

-19 Friedlander M, Brooks PC, Shaffer RW, Kincaid CM, Varner JA, Cheresh DA: Definition of two angiogenic pathways by distinct alpha v integrins. Science 1995;270:1500-1502.

20 Shono T, Mochizuki Y, Kanetake H, Kanda S: Inhibition of FGF-2-mediated chemotaxis of murine brain capillary endothelial cells by cyclic RGDfV peptide through blocking the redistribution of c-Src into focal adhesions. Exp Cell Res 2001;268:169-178.

-21 Laemmli UK: Cleavage of structural proteins during the assembly of the head of bacteriophage T4. Nature 1970;227:680-685.

$>22$ Lal A, Haynes SR, Gorospe M: Clean Western blot signals from immunoprecipitated samples. Mol Cell Probes 2005;19:385-388.
23 Hanke JH, Gardner JP, Dow RL, Changelian PS, Brissette WH, Weringer EJ, et al: Discovery of a novel, potent, and Src family-selective tyrosine kinase inhibitor. Study of Lck- and FynT-dependent $\mathrm{T}$ cell activation. J Biol Chem 1996;271:695-701.

24 Waltenberger J, Uecker A, Kroll J, Frank H, Mayr U, Bjorge JD, et al: A dual inhibitor of platelet-derived growth factor beta-receptor and Src kinase activity potently interferes with motogenic and mitogenic responses to PDGF in vascular smooth muscle cells. A novel candidate for prevention of vascular remodeling. Circ Res 1999;85:12-22.

25 Pfaff M, Tangemann K, Muller B, Gurrath M, Muller G, Kessler H, et al: Selective recognition of cyclic RGD peptides of NMR defined conformation by alpha IIb beta 3 , alpha V beta 3 , and alpha 5 beta 1 integrins. J Biol Chem 1994:269:20233-20238.

26 Katsumi A, Orr AW, Tzima E, Schwartz MA: Integrins in mechanotransduction. J Biol Chem 2004;279:12001-12004.

27 Martinez-Lemus LA, Crow T, Davis MJ, Meininger GA: alphavbeta3- and alpha5beta1-integrin blockade inhibits myogenic constriction of skeletal muscle resistance arterioles. Am J Physiol Heart Circ Physiol 2005; 289:H322-H329.

28 Wilson E, Sudhir K, Ives HE: Mechanical strain of rat vascular smooth muscle cells is sensed by specific extracellular matrix/integrin interactions. J Clin Invest 1995;96:23642372.

29 Davis MJ, Hill MA: Signaling mechanisms underlying the vascular myogenic response. Physiol Rev 1999;79:387-423.

30 Heerkens EH, Izzard AS, Heagerty AM: Integrins, vascular remodeling, and hypertension. Hypertension 2007;49:1-4.

31 Thybo NK, Korsgaard N, Mulvany MJ: Morphology and function of mesenteric resistance arteries in transgenic rats with low-renin hypertension. J Hypertens 1992;10:11911196.
2 Baumbach GL, Sigmund CD, Faraci FM: Cerebral arteriolar structure in mice overexpressing human renin and angiotensinogen. Hypertension 2003;41:50-55.

33 Brosnan MJ, Devlin AM, Clark JS, Mullins JJ, Dominiczak AF: Different effects of antihypertensive agents on cardiac and vascular hypertrophy in the transgenic rat line TGR(mRen2)27. Am J Hypertens 1999;12: 724-731.

34 Polte TR, Naftilan AJ, Hanks SK: Focal adhesion kinase is abundant in developing blood vessels and elevation of its phosphotyrosine content in vascular smooth muscle cells is a rapid response to angiotensin II. J Cell Biochem 1994;55:106-119.

-35 Haendeler J, Berk BC: Angiotensin II mediated signal transduction. Important role of tyrosine kinases. Regul Pept 2000;95:1-7.

-36 Tahiliani PD, Singh L, Auer KL, LaFlamme SE: The role of conserved amino acid motifs within the integrin beta3 cytoplasmic domain in triggering focal adhesion kinase phosphorylation. J Biol Chem 1997;272:7892-7898.

37 Varadarajulu J, Laser M, Hupp M, Wu R, Hauck CR: Targeting of alpha(v) integrins interferes with FAK activation and smooth muscle cell migration and invasion. Biochem Biophys Res Commun 2005;331:404-412.

38 Xia H, Nho RS, Kahm J, Kleidon J, Henke CA: Focal adhesion kinase is upstream of phosphatidylinositol 3-kinase/Akt in regulating fibroblast survival in response to contraction of type I collagen matrices via a beta 1 integrin viability signaling pathway. J Biol Chem 2004; 279:33024-33034.

39 Ma H, Calderon TM, Kessel T, Ashton AW, Berman JW: Mechanisms of hepatocyte growth factor-mediated vascular smooth muscle cell migration. Circ Res 2003;93: 1066-1073. 